\title{
Does preoperative hyponatremia potentiate the effects of left ventricular dysfunction on mortality after cardiac surgery?
}

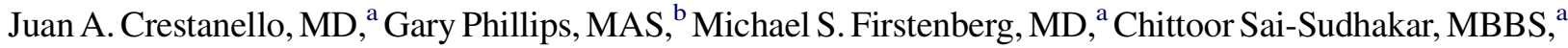 \\ John Sirak, MD, ${ }^{a}$ Robert Higgins, $M D,{ }^{a}$ and William T. Abraham, $\mathrm{MD}^{\mathrm{c}}$
}

Objective: Left ventricular dysfunction and preoperative hyponatremia are associated with adverse outcomes after cardiac surgery. However, the interactions between them are unknown. Thus, we evaluated the interaction of low left ventricular ejection fraction $(<40 \%)$ and preoperative hyponatremia $(\mathrm{Na}<135 \mathrm{mEq} / \mathrm{L})$ with morbidity and mortality after cardiac surgery.

Methods: The interaction of hyponatremia and ejection fraction with hospital complications, length of stay, and mortality was analyzed using logistic and Cox regression analysis in 2247 patients who underwent cardiac surgery between 2005 and 2008 at The Ohio State University Wexner Medical Center.

Results: Of the patients, $68.5 \%$ had normal ejection fraction. Hyponatremia was present in $18 \%$ of patients with normal ejection fraction and $35 \%$ of patients with low ejection fraction. Hyponatremic patients had higher rates of New York Heart Association class III and IV, more comorbidities, and higher Society of Thoracic Surgeons score and European System for Cardiac Operative Risk Evaluation irrespectively of their ejection fraction. The correlation between preoperative sodium and ejection fraction was weak $\left(r^{2}=0.04\right)$. Hyponatremia increased the rate of postoperative complications and hospital stay, and decreased 1- and 3-year survivals in patients with both normal and low ejection fraction. Hyponatremia was independently associated with longer hospital stay for normal ejection fraction (multiplier, 1.18; confidence interval, 1.09-1.27; $P<.001$ ) and low ejection fraction (multiplier, 1.10; confidence interval, 1.0-1.21; $P=.05$ ), increased need for dialysis for normal ejection fraction (odds ratio, 2.16; confidence interval, 1.08-4.32; $P=.03$ ), and increased risk of mortality for normal ejection fraction (hazard ratio, 1.56; confidence interval, 1.20-2.05; $P=.001$ ), but not for patients with low ejection fraction (hazard ratio, 1.21; confidence interval, $0.89-1.65 ; P=.21$ ).

Conclusions: Hyponatremia is more common in patients with low ejection fraction. Although preoperative hyponatremia is independently associated with adverse outcomes in patients with normal ejection fraction, an association with adverse outcomes in patients with low ejection fraction was not demonstrated. (J Thorac Cardiovasc Surg 2013;145:1589-94)

\section{Supplemental material is available online.}

Hyponatremia is a common electrolyte abnormality in critically ill patients and adversely affects outcomes. ${ }^{1,2}$ It is particularly common in patients with congestive heart

\footnotetext{
From the Division of Cardiac Surgery, ${ }^{\mathrm{a}}$ Center for Biostatistics, ${ }^{\mathrm{b}}$ and Division of Cardiovascular Medicine, ${ }^{\mathrm{c}}$ The Ohio State University, Wexner Medical Center, Columbus, Ohio.

This work was supported by a research grant from Biogen Idec (Weston, Mass).

Disclosures: Dr Crestanello received a research grant from Biogen Idec, and Dr Abraham received a research grant and consulting fees from Biogen Idec. All other authors have nothing to disclose with regard to commercial support.

Presented at the American Heart Association 2011 Scientific Sessions, Orlando, Florida, November 12-16, 2011.

Received for publication Feb 28, 2012; revisions received Nov 15, 2012; accepted for publication Dec 10, 2012; available ahead of print April 8, 2013.

Address for reprints: Juan A. Crestanello, MD, N-816 Doan Hall, 410 West 10th Ave, Columbus, OH 43210 (E-mail: juan.crestanello@osumc.edu).

$0022-5223 / \$ 36.00$

Copyright (c) 2013 by The American Association for Thoracic Surgery

http://dx.doi.org/10.1016/j.jtcvs.2012.12.093
}

failure, in whom its prevalence and severity correlate with the prevalence and severity of heart failure and ventricular dysfunction. ${ }^{3-5}$ We previously demonstrated that preoperative hyponatremia is common before cardiac surgery and is an independent risk factor for mortality, prolonged hospitalization, and postoperative complications after cardiac surgery. ${ }^{6}$ Left ventricular (LV) dysfunction is also an independent predictor of poor outcomes after cardiac surgery. It has been associated with increased morbidity and mortality after coronary artery bypass surgery, valve surgery, combined bypass and valve surgery, and other cardiac surgery procedures. ${ }^{7-10}$

The interaction between preoperative hyponatremia and LV dysfunction and its effects on outcomes after cardiac surgery have not been evaluated. We hypothesize that hyponatremia may potentiate the adverse effect of LV dysfunction in outcomes after cardiac surgery. We retrospectively studied 2247 patients undergoing cardiac surgery to determine the prevalence of preoperative hyponatremia in patients with LV dysfunction before cardiac surgery and the interaction between preoperative hyponatremia and LV 


\section{Abbreviations and Acronyms \\ $\mathrm{CABG}=$ coronary artery bypass grafting \\ $\mathrm{CI}=$ confidence interval \\ $\mathrm{EF}=$ ejection fraction \\ $\mathrm{LV}=$ left ventricular \\ NYHA $=$ New York Heart Association \\ STS $=$ Society of Thoracic Surgeons}

dysfunction on hospital complications and on short- and long-term mortality after cardiac surgery.

\section{MATERIALS AND METHODS}

From January 2005 to December 2008, 2785 patients underwent cardiac surgery procedures at The Ohio State University Wexner Medical Center. Preoperative serum sodium and LV ejection fraction (EF) determinations were available in 2247 patients. These patients constitute the study population. Hyponatremia was defined as serum sodium less than $135 \mathrm{mEq} / \mathrm{L}$ in any of the 3 most recent daily sodium determinations performed within 30 days before surgery. Systolic LV dysfunction was defined as an LV EF less than $40 \%$. LV EF was assessed by ventriculogram in $63.6 \%$ of patients, echocardiogram in $35.5 \%$ of patients, and other methods in $0.9 \%$ of patients. Patients were divided into 4 groups according to EF and hyponatremia status: (1) low $\mathrm{EF}(\mathrm{EF}<40 \%)$ and normonatremia $(\mathrm{Na} \geq 135 \mathrm{mEq} / \mathrm{L})$ group, (2) low $\mathrm{EF}(\mathrm{EF}<40 \%)$ and hyponatremia $(\mathrm{Na}<135 \mathrm{mEq} / \mathrm{L})$ group, (3) normal $\mathrm{EF}(\mathrm{EF} \geq 40 \%$ ) and normonatremia ( $\mathrm{Na} \geq 135 \mathrm{mEq} / \mathrm{L})$ group, and (4) normal $\mathrm{EF}(\mathrm{EF} \geq 40 \%)$ and hyponatremia $(\mathrm{Na}<135 \mathrm{mEq} / \mathrm{L})$ group.

Data from the institution electronic medical records, the institution Society of Thoracic Surgeons (STS) database, and the Social Security Death Index were analyzed. Outcomes analyzed included early and late mortality, postoperative length of hospital stay, incidence of STS-defined complications, ${ }^{11,12}$ and need for perioperative blood transfusions. Outcomes definitions are listed in Appendix E1. The Ohio State University Wexner Medical Center Institutional Review Board approved this study and granted waiver of Health Insurance Portability and Accountability Act research authorization.

\section{Statistical Analysis}

Baseline characteristics are presented as means, standard deviations, medians, and interquartile ranges for continuous variables. Categoric variables are presented as frequencies. Baseline characteristics were compared using Pearson's chi-square test for categoric variables and Kruskal-Wallis for continuous variables. Regression models were developed using a risk factor modeling approach to determine which covariates were included in the model. ${ }^{13,14}$ Covariates that acted as a confounder or an effect modifier were included (Appendix E2). Logistic regression was used to find the association between hyponatremia and EF status with STS complications. Logistic regression was used when the outcome was early mortality. Linear regression was used when the outcome was log-transformed hospital length of stay. Cox proportional hazard regression was used to find the association of late and overall mortality with hyponatremia and EF status. Serum sodium was treated as both a continuous and a dichotomous variable. Survival was estimated by the Kaplan-Meier method, and differences in survival were compared using the log-rank test. All analyses were conducted using Stata 11.1 (StataCorp LP, College Station, Tex).

\section{RESULTS}

Preoperative hyponatremia was present in 527 patients $(23.5 \%)$ before cardiac surgery. The distribution of preoperative EF is shown in Figure E1. Low EF was present in 707 patients $(31.5 \%)$. A total of 282 patients $(18.3 \%)$ in the normal EF group and 245 patients (34.6\%) in the low EF group had preoperative hyponatremia. Hyponatremic and normonatremic patients had clinically similar EF within their EF group (Table 1). The correlation between preoperative serum sodium and preoperative EF was weak $\left(r^{2}=0.04\right)$.

Demographics, clinical, and surgical characteristics are shown in Table 1. Compared with those with normal EF, patients with low EF were younger and more often male. They more often had congestive heart failure and a higher prevalence of myocardial infarction, chronic obstructive pulmonary disease, stroke, peripheral vascular disease, diabetes, renal dysfunction, and previous cardiac surgery. These comorbidities resulted in higher predicted risk of mortality as determined by European System for Cardiac Operative Risk Evaluation and STS Predicted Risk of Mortality. Hyponatremic patients had a higher rate of New York Heart Association (NYHA) functional class III and IV, higher rate of previous cardiac surgery, and higher European System for Cardiac Operative Risk Evaluation irrespectively of their EF status.

Isolated coronary artery bypass grafting $(\mathrm{CABG})$ and isolated valve surgery were more common in the normal $\mathrm{EF}$ group, and combined CABG-valve, ventricular assist device, and other cardiac surgery procedures were more common in the low EF group. Cardiopulmonary bypass time and crossclamp times were not clinically different among the groups.

Table 2 shows unadjusted clinical outcomes. The rate of postoperative complications (operative, infectious, pulmonary, renal failure, need for dialysis, and other complications) was higher in the low EF group. Hyponatremia increased the incidence of operative, pulmonary, and renal complications compared with normonatremia in both the normal and low EF groups. Low EF was associated with longer hospital stay. Hyponatremia increased the length of hospital stay in both the normal and low EF groups. Early and late mortality were increased in the low EF group compared with the normal EF group (early mortality: $8.5 \%$ vs $3.9 \%$ in normal EF, $P<.001$; late mortality: $20.4 \%$ vs $13.2 \%$ in normal EF, $P<.001)$. Hyponatremia further increased early and late mortality in both the normal and low EF groups (Table 2). Kaplan-Meier survival curves by hyponatremia and EF status are shown in Figure 1. Median follow-up was 1.31 years (interquartile range, 0.22.58). Hyponatremia decreased the estimated 1- and 3-year survivals in both the normal EF and low EF groups compared with normonatremia.

Figure E2 shows a univariate analysis of the clinical outcomes. Hyponatremia increased the odds of operative, pulmonary, and renal complications; renal failure requiring dialysis; length of hospital stay; and early mortality in both the low and normal EF cohorts. It also increased the hazard of late and overall mortality in both groups. After adjusting for baseline and procedural variables (Figure 2), 
TABLE 1. Patients' clinical characteristics and operative variables stratified according to preoperative left ventricular ejection fraction and serum sodium

\begin{tabular}{|c|c|c|c|c|c|c|c|}
\hline & \multicolumn{3}{|c|}{ Low EF N $=707$} & \multicolumn{3}{|c|}{ Normal EF $N=1540$} & \multirow[b]{2}{*}{$\begin{array}{l}P \text { norma } \\
\text { vs low EI }\end{array}$} \\
\hline & $\mathrm{Na} \geq 135 \mathrm{mEq} / \mathrm{L}$ & $\mathrm{Na}<135 \mathrm{mEq} / \mathrm{L}$ & $\begin{array}{c}P \mathrm{Na}<135 \\
\mathrm{mEq} / \mathrm{L} \\
\text { vs } \mathrm{Na} \geq 135 \\
\mathrm{mEq} / \mathrm{L}\end{array}$ & $\mathrm{Na} \geq 135 \mathrm{mEq} / \mathrm{L}$ & $\mathrm{Na}<135 \mathrm{mEq} / \mathrm{L}$ & $\begin{array}{c}P \mathrm{Na}<135 \\
\mathrm{mEq} / \mathrm{L} \\
\text { vs } \mathrm{Na} \geq 135 \\
\mathrm{mEq} / \mathrm{L}\end{array}$ & \\
\hline $\mathrm{N}$ & 462 & 245 & - & 1258 & 282 & - & - \\
\hline Mean $\mathrm{Na} \pm \mathrm{SD}(\mathrm{mEq} / \mathrm{L})$ & $138.5 \pm 2.2$ & $133.2 \pm 3.0$ & $<.001$ & $138.7 \pm 1.8$ & $133.9 \pm 2.4$ & $<.001$ & $<.001$ \\
\hline Age (y) & $61 \pm 12$ & $59 \pm 12$ & .004 & $62 \pm 13$ & $63 \pm 14$ & .117 & .004 \\
\hline Male gender, $\mathrm{N}(\%)$ & $324(70.1)$ & $175(71.4)$ & .718 & $825(65.6)$ & $173(61.4)$ & .179 & .007 \\
\hline White race, $\mathrm{N}(\%)$ & $383(82.9)$ & $196(80.0)$ & .341 & $1094(87.0)$ & $236(83.7)$ & .147 & .006 \\
\hline $\mathrm{LV}$ EF $(\%$, mean $\pm \mathrm{SD})$ & $26 \pm 8$ & $24 \pm 9$ & $<.001$ & $53 \pm 7$ & $53 \pm 8$ & .067 & N/A \\
\hline $\begin{array}{l}\text { Mean PA pressure } \\
\quad(\mathrm{mm} \mathrm{Hg}, \text { mean } \pm \mathrm{SD})\end{array}$ & $32.3 \pm 10.8$ & $32.8 \pm 9.6$ & .380 & $27.0 \pm 9.5$ & $32.0 \pm 13.4$ & $<.001$ & $<.001$ \\
\hline $\operatorname{GFR}\left(\mathrm{mg} / \mathrm{min} / 1.73 \mathrm{~m}^{2}\right)$ & $69 \pm 29$ & $64 \pm 29$ & .094 & $73 \pm 26$ & $74 \pm 32$ & .754 & .001 \\
\hline NYHA class III-IV $(\%)$ & $356(77.1)$ & $204(83.4)$ & .050 & $689(54.8)$ & $192(68.2)$ & $<.001$ & $<.001$ \\
\hline Diabetes, N (\%) & $203(43.9)$ & $115(46.9)$ & .446 & $423(33.6)$ & $134(47.5)$ & $<.001$ & $<.001$ \\
\hline Hypertension, $\mathrm{N}(\%)$ & $393(85.1)$ & $196(80.0)$ & .086 & $1035(82.3)$ & $249(88.3)$ & .014 & .968 \\
\hline $\begin{array}{l}\text { Renal failure on dialysis, } \\
\quad \mathrm{N}(\%)\end{array}$ & $57(12.3)$ & $42(17.0)$ & .260 & $86(6.8)$ & $30(10.7)$ & .150 & .002 \\
\hline Previous MI, N (\%) & $286(61.9)$ & $152(62.0)$ & .972 & $452(35.9)$ & $116(41.1)$ & .102 & $<.001$ \\
\hline COPD, N (\%) & $147(31.8)$ & $99(40.4)$ & .022 & $292(23.2)$ & $84(29.7)$ & .020 & $<.001$ \\
\hline Stroke, N (\%) & $75(16.2)$ & $42(17.2)$ & .864 & $157(12.5)$ & $37(13.2)$ & .864 & .017 \\
\hline PVD, N (\%) & $94(20.4)$ & $58(23.7)$ & .367 & $199(15.8)$ & $59(20.9)$ & .038 & .009 \\
\hline $\begin{array}{l}\text { Previous cardiac surgery, } \\
\text { N }(\%)\end{array}$ & $59(12.8)$ & $47(19.2)$ & .023 & $127(10.1)$ & $45(16.0)$ & .005 & .011 \\
\hline euroSCORE, \% (IQR) & $29(12-54)$ & $46(23-71)$ & $<.001$ & $10(4-27)$ & $19(6-45)$ & $<.001$ & $<.001$ \\
\hline STS PROM, \% (IQR) & $2.68(1.17-5.99)$ & $2.95(1.54-6.66)$ & .337 & $1.23(0.62-2.83)$ & $1.53(0.69-4.69)$ & .011 & $<.001$ \\
\hline \multicolumn{8}{|l|}{ Surgical characteristics } \\
\hline $\mathrm{CPB}$ time, $\min \pm \mathrm{SD}$ & $130 \pm 53$ & $125 \pm 67$ & .128 & $117 \pm 57$ & $126.0 \pm 64$ & .134 & $<.001$ \\
\hline $\begin{array}{l}\text { Crossclamp time, } \\
\min \pm \mathrm{SD}\end{array}$ & $98 \pm 39$ & $95 \pm 45$ & .307 & $91 \pm 43$ & $96 \pm 49$ & .485 & .001 \\
\hline CABG only, N (\%) & $179(38.7)$ & $65(26.5)$ & .001 & $629(50.0)$ & $130(46.1)$ & .236 & $<.001$ \\
\hline Valve surgery only, $\mathrm{N}(\%)$ & $52(11.3)$ & $23(9.4)$ & .443 & $182(14.5)$ & $46(16.3)$ & .431 & .007 \\
\hline CABG valve, $\mathrm{N}(\%)$ & $100(21.6)$ & $33(13.5)$ & .008 & $125(9.9)$ & $42(14.9)$ & .016 & $<.001$ \\
\hline VAD surgery only, $\mathrm{N}(\%)$ & $11(2.4)$ & $29(11.8)$ & $<.001$ & $2(0.2)$ & $0(0)$ & .503 & $<.001$ \\
\hline Other surgery, $\mathrm{N}(\%)$ & $120(26)$ & $22(9.0)$ & $<.001$ & $320(25.4)$ & $64(22.7)$ & .33 & .013 \\
\hline
\end{tabular}

$E F$, Ejection fraction; $S D$, standard deviation; $L V$, left ventricular; $P A$, pulmonary artery; $G F R$, glomerular filtration rate; $N Y H A$, New York Heart Association; $M I$, myocardial infarction; COPD, chronic obstructive pulmonary disease; $P V D$, peripheral vascular disease; euroSCORE, European System for Cardiac Operative Risk Evaluation; IQR, interquartile range; $S T S$ PROM, Society of Thoracic Surgeons Predicted Risk of Mortality; $C P B$, cardiopulmonary bypass; $C A B G$, coronary artery bypass grafting; $V A D$, ventricular assist device; $N / A$, not available.

hyponatremia was associated with increased length of hospital stay for normal EF (multiplier, 1.18; confidence interval [CI], 1.09-1.27; $P<.001$ ) and low EF (multiplier, 1.10; CI, $1.00-1.21 ; P=.05$ ) cohorts. The odds of dialysis (odds ratio, 2.16; CI, 1.08-4.32; $P=.03$ ) and early mortality (odds ratio, $1.93 ; \mathrm{CI}, 1.00-3.69 ; P=.047)$, and the hazard of late (hazard ratio, $1.54 ; \mathrm{CI}, 1.13-2.11 ; P=.008)$ and overall mortality (hazard ratio, 1.56; CI, 1.20-2.05; $P=.01$ ) were increased by hyponatremia for patients with normal EF. After adjusting for baseline and operative variables, hyponatremia did not increase early, late, or overall mortality in the low EF cohort.

Figure 3 displays the adjusted overall mortality in function of serum sodium as a continuous variable for low and normal EF cohorts. The adjusted mortality rate increases with the severity of preoperative hyponatremia in those with normal EF but not in those with low EF.

\section{DISCUSSION}

This study demonstrated that (1) preoperative hyponatremia is approximately twice as frequent in patients with low $\mathrm{EF}$ than in patients with normal EF, (2) there is not a strong direct correlation between $\mathrm{EF}$ and preoperative serum sodium, (3) preoperative hyponatremia increases the unadjusted rate of postoperative complications and mortality and decreases long-term survival for patients with normal EF and patients with low EF, and (4) preoperative hyponatremia increases the hazard of mortality for patients with normal EF. However, preoperative hyponatremia was not 
TABLE 2. Unadjusted clinical outcomes stratified by preoperative left ventricular ejection fraction and serum sodium

\begin{tabular}{|c|c|c|c|c|c|c|c|}
\hline & \multicolumn{3}{|c|}{ Low EF N = 707 } & \multicolumn{3}{|c|}{ Normal EF N = 1540} & \multirow[b]{2}{*}{$\begin{array}{l}P \text { normal } \\
\text { vs low EF }\end{array}$} \\
\hline & $\mathrm{Na} \geq 135 \mathrm{mEq} / \mathrm{L}$ & $\mathrm{Na}<135 \mathrm{mEq} / \mathrm{L}$ & $\begin{array}{c}P \mathrm{Na}<135 \\
\mathrm{mEq} / \mathrm{L} \\
\text { vs Na } \geq 135 \\
\mathrm{mEq} / \mathrm{L} \\
\end{array}$ & $\mathrm{Na} \geq 135 \mathrm{mEq} / \mathrm{L}$ & $\mathrm{Na}<135 \mathrm{mEq} / \mathrm{L}$ & $\begin{array}{c}P \mathrm{Na}<135 \\
\mathrm{mEq} / \mathrm{L} \\
\text { vs } \mathrm{Na} \geq 135 \\
\mathrm{mEq} / \mathrm{L} \\
\end{array}$ & \\
\hline $\mathrm{N}$ & 462 & 245 & - & 1258 & 282 & - & - \\
\hline \multicolumn{8}{|l|}{ Complications } \\
\hline Operative, N (\%) & $57(12.3)$ & $55(22.5)$ & $<.001$ & $103(8.2)$ & $35(12.4)$ & .0250 & $<.001$ \\
\hline Infectious, $N(\%)$ & $32(6.9)$ & $20(8.2)$ & .549 & $36(2.9)$ & $17(6.0)$ & .0110 & $<.001$ \\
\hline Pulmonary, N (\%) & $138(29.9)$ & $117(47.8)$ & $<.001$ & $283(22.5)$ & $92(32.6)$ & $<.001$ & $<.001$ \\
\hline Neurologic, N (\%) & $12(2.6)$ & $11(4.5)$ & .177 & $34(2.7)$ & $8(2.8)$ & .9000 & .49 \\
\hline Renal failure, N (\%) & $39(8.4)$ & $37(15.1)$ & .007 & $73(5.8)$ & $39(13.8)$ & $<.001$ & .006 \\
\hline Dialysis, N (\%) & $24(5.2)$ & $24(9.8)$ & .021 & $39(3.1)$ & $25(8.9)$ & $<.001$ & .008 \\
\hline Other, N (\%) & $134(29.0)$ & $72(29.4)$ & .915 & $328(26.1)$ & $90(31.9)$ & .0460 & .327 \\
\hline Blood transfusion & $219(47.5)$ & $142(57.9)$ & .009 & $513(40.8)$ & $162(57.5)$ & $<.001$ & .002 \\
\hline Hospital LOS, d (IQR) & $11.5(7.61-17.9)$ & $18.2(10.9-12.8)$ & $<.001$ & $8.28(6.12-12.8)$ & $11.6(7.72-19.0)$ & $<.001$ & $<.001$ \\
\hline \multicolumn{8}{|l|}{ Mortality } \\
\hline Early mortality, N (\%) & $30(6.5)$ & $30(12.2)$ & .009 & $40(3.2)$ & $20(7.1)$ & .0020 & $<.001$ \\
\hline Late mortality, N (\%) & $84(18.2)$ & $60(24.5)$ & .047 & $146(11.6)$ & $57(20.2)$ & $<.001$ & $<.001$ \\
\hline
\end{tabular}

Early mortality: any death during the hospitalization for surgery or after discharge from the hospital, but within 30 days of the procedure. Late mortality: any death after discharge from the hospital and more than 30 days from the operative procedure. For definitions of complications see Appendix E1. EF, Ejection fraction; $L O S$, length of stay; $I Q R$, interquartile range.

independently associated with an increased hazard of mortality in those with low EF.

Hyponatremia is common in patients with heart failure and patients with LV dysfunction. ${ }^{3-5}$ The prevalence of hyponatremia in heart failure ranges from $5 \%$ in outpatients to $27 \%$ in hospitalized patients. ${ }^{1,3-5}$ The present study reported for the first time the prevalence of preoperative hyponatremia in patients with normal and decreased LV function in need of cardiac surgery. The overall prevalence was similar to that of patients hospitalized with heart failure., ${ }^{4,15}$ However, the prevalence of hyponatremia in patients with low EF was twice as high as that in patients with normal EF. The high prevalence of preoperative hyponatremia in patients with LV dysfunction waiting for cardiac surgery reflects a more advanced stage of cardiac disease and higher prevalence of associated comorbidities (Table 1).

Previous studies have demonstrated that hyponatremia is a marker of disease severity in congestive heart failure with and without depressed LV EF. ${ }^{1,3,5,16}$ The current study did not find a strong correlation between serum sodium and LV EF. In addition, EF was similar in patients with and

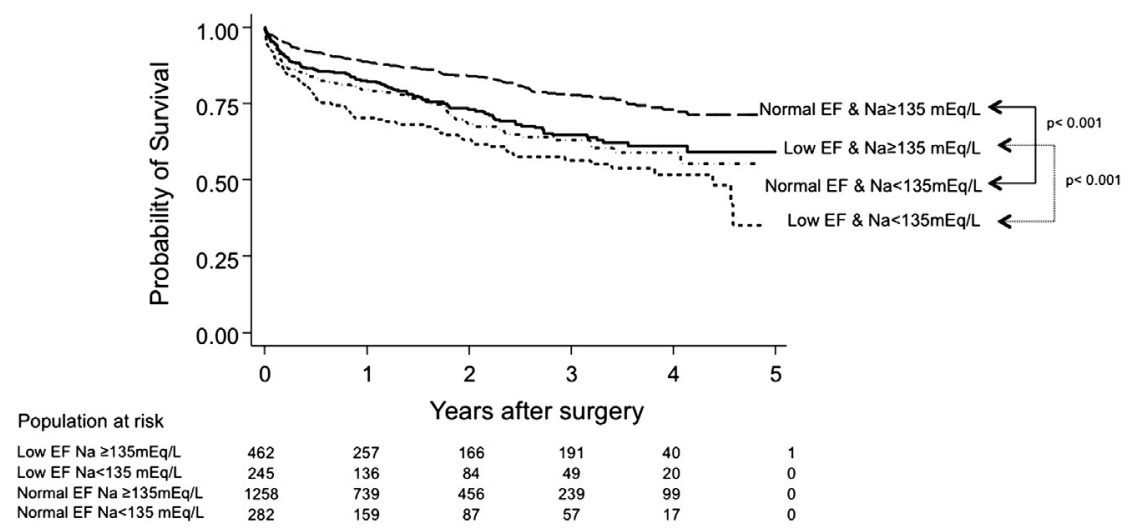

FIGURE 1. Kaplan-Meier survival curve stratified according to preoperative sodium level and EF. Hyponatremia decreased 1- and 3-year unadjusted survivals for both the low and normal EF cohorts (log-rank $P<.001$ ). One-year survival for the low EF cohort was $82 \%(95 \% \mathrm{CI}, 78-86)$ for Na $135 \mathrm{mEq} / \mathrm{L}$ or greater versus $70 \%(95 \% \mathrm{CI}, 63-75)$ for Na less than $135 \mathrm{mEq} / \mathrm{L}$. One-year survival for the normal EF cohort was $88 \%$ (95\% CI, 86-90) for Na 135 $\mathrm{mEq} / \mathrm{L}$ or greater versus $79 \%$ (95\% CI, 74-84) for Na less than $135 \mathrm{mEq} / \mathrm{L}$. Three-year survival for the low EF cohort was $64 \%(95 \% \mathrm{CI}, 58-70)$ for Na $135 \mathrm{mEq} / \mathrm{L}$ or greater versus $56 \%(95 \%$ CI, 48-63) for Na less than $135 \mathrm{mEq} / \mathrm{L}$. Three-year survival for the normal EF cohort was $77 \%$ (95\% CI, 74-80) for Na 135 $\mathrm{mEq} / \mathrm{L}$ or greater versus $62 \%(95 \% \mathrm{CI}, 55-69)$ for $\mathrm{Na}$ less than $135 \mathrm{mEq} / \mathrm{L}$. EF, Ejection fraction; $C I$, confidence interval. 


\section{Low EF}

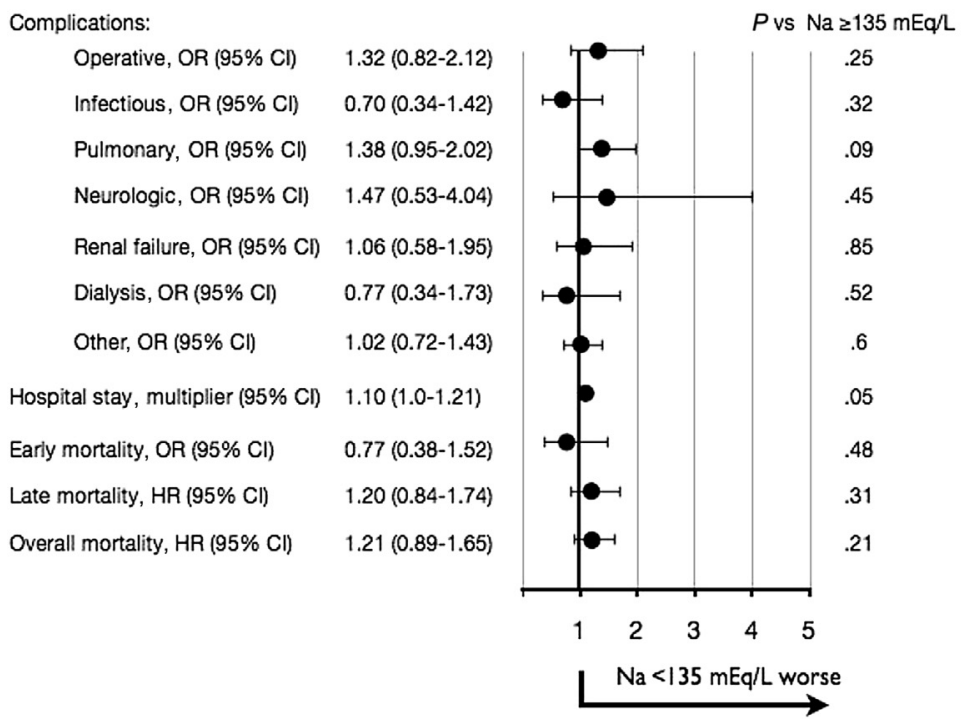

Normal EF

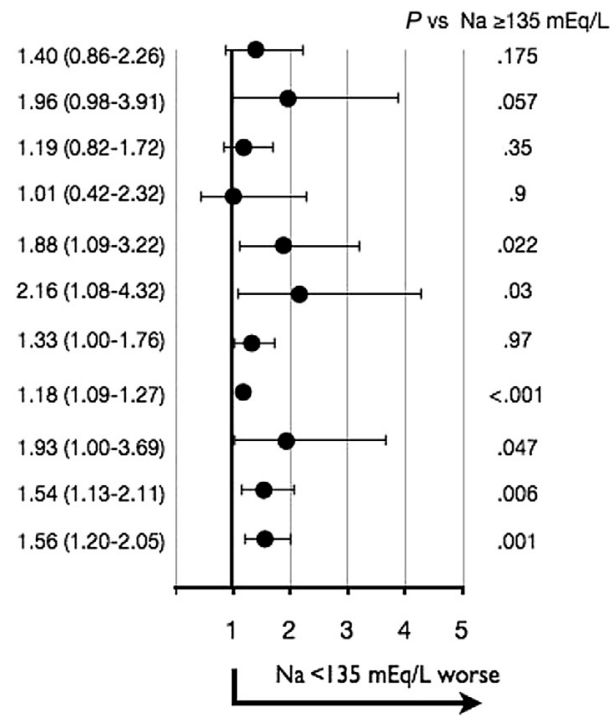

FIGURE 2. Effect of hyponatremia on clinical outcomes of patients with low and normal ejection fractions by multivariable regression analysis. See Appendix E2 for covariates included in the analysis. EF, Ejection fraction; $O R$, odds ratio; $C I$, confidence interval; $H R$, hazard ratio.

without hyponatremia within each cohort of the current study. These findings are not surprising because the most critical factors in the development of hyponatremia in heart failure is the reduction in cardiac output and the degree of neurohormonal activation. ${ }^{17}$ Many patients with decreased EF are capable of maintaining cardiac output secondary to a compensatory increase in LV end-diastolic volume. ${ }^{18}$ This study quantified heart failure severity by evaluating NYHA class. Compared with normonatremia, hyponatremic patients had a higher prevalence of NYHA class III and IV symptoms regardless of their EF. Therefore, preoperative hyponatremia correlates more with the overall severity of heart failure than with $\mathrm{EF}$ alone.

Hyponatremia has been associated with adverse outcomes in multiple clinical settings. ${ }^{1-6}$ However, the value of preoperative hyponatremia to predict outcomes in patients undergoing cardiac surgery has not been established. Conversely, LV dysfunction is a well-established risk factor associated with adverse outcomes after cardiac surgery procedures. ${ }^{7-10} \mathrm{LV}$ dysfunction has been associated with increased risk of early and late mortality after CABG, aortic and mitral valve surgery, and combined valve CABG. $^{7-10}$ The present study demonstrated increased early and late mortality in a large group of patients with low LV EF undergoing a variety of cardiac surgery procedures that are representative of the surgical practice in this country. We expected that the coexistence of preoperative hyponatremia with LV dysfunction may accentuate the adverse effects of LV dysfunction on outcomes. In the OPTIMIZE-HF registry, which included 47,000 patients hospitalized with heart failure, the combination of hyponatremia and LV dysfunction increased the unadjusted rates of in hospital mortality, length of hospital stay, rehospitalization for heart failure, and long-term mortality compared with hyponatremia alone. ${ }^{3}$ In addition, hyponatremia was independently associated with an increased risk of mortality in patients with normal EF and patients with low EF. In the present study, preoperative hyponatremia was associated by univariate analysis with postoperative complications, length of hospital stay, and early and late mortality in both low and normal EF cohorts. However, hyponatremia was only independently associated with adverse outcomes in patients with preserved LV systolic function. The failure to observe an independent effect on mortality in patients with low EF is puzzling and led to the generation of 2 hypotheses to explain this finding: (1) The interaction of the therapeutic intervention (cardiac surgery) in outcomes may disrupt that association. Contrary to patients with congestive heart failure treated medically, cardiac surgery procedures may correct or ameliorate heart failure and disrupt the independent effect of hyponatremia in outcomes. (2) Hyponatremia might behave as a marker of disease severity and neurohormonal activation in low EF, whereas in patients with normal EF, it has an independent and direct deleterious effect on myocardial function that predisposes those patients to poor outcomes after surgery. Elucidation of these hypotheses will require additional studies specifically designed to address them.

\section{Study Limitations}

First, this was a single-center retrospective study, and the results should be interpreted within such context. Second, despite the overall large population included in the study, there were only 245 patients $(10.9 \%)$ with hyponatremia and low EF. Third, the preoperative EF was determined by a variety of 

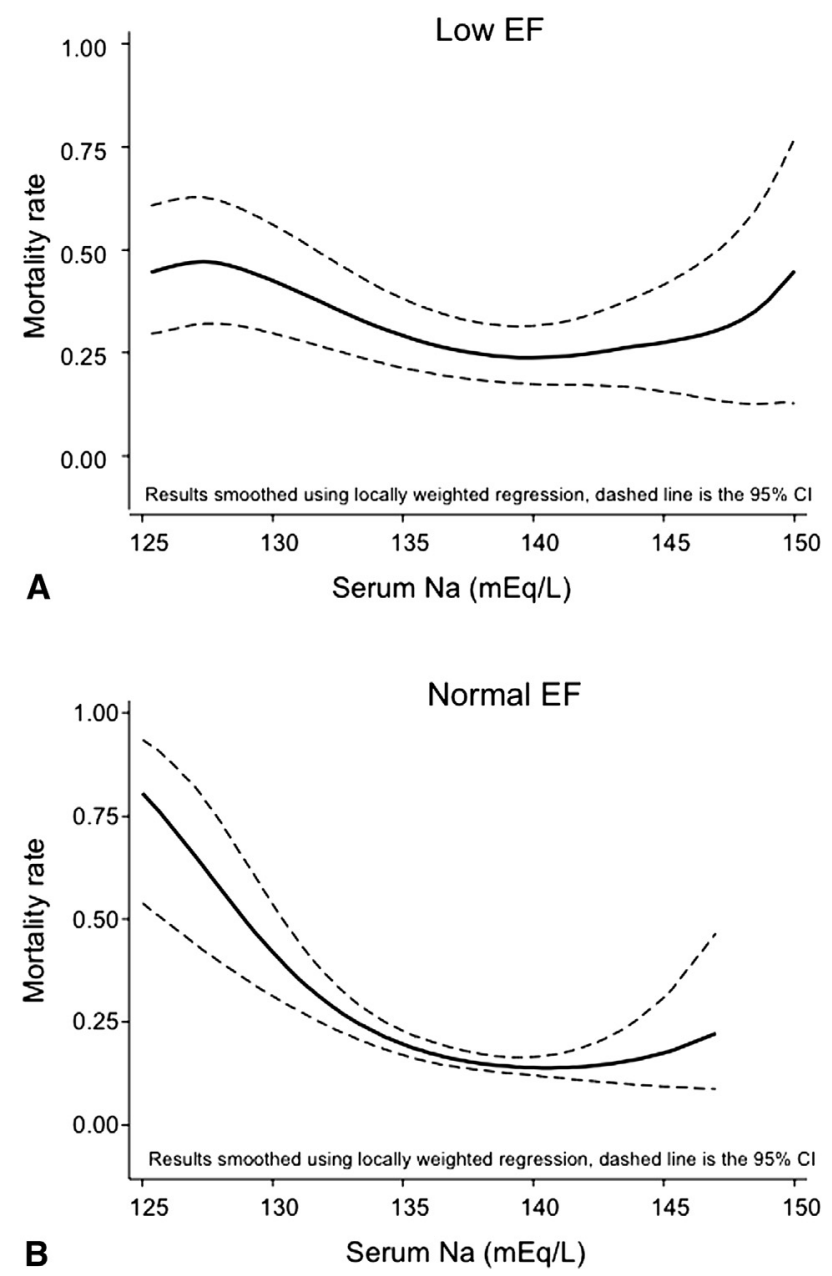

FIGURE 3. Association between preoperative serum sodium and adjusted overall mortality for patients with low EF (A) and normal EF (B). The adjusted mortality rate increases with the severity of preoperative hyponatremia in the normal EF cohort (B) but not in the low EF cohort (A). Dashed lines represent 95\% CI. See Appendix E2 for covariates included in the model. $E F$, Ejection fraction; $C I$, confidence interval.

methods (echocardiogram, ventriculogram, and others) during standard clinical care, which may have resulted in considerable variability in EF measurements. Fourth, low EF was defined as an EF less than $40 \%$. Therefore, patients with moderate and severe LV dysfunction were included in the low EF group. It is possible that preoperative hyponatremia may have different effects on outcomes in those groups. Fifth, serum sodium levels were not adjusted for glucose levels. Hyperglycemia leads to hyponatremia by mechanisms different than those responsible for hyponatremia in heart failure. It is unknown whether hyponatremia secondary to hyperglycemia has any adverse effects in outcomes. ${ }^{19}$

\section{CONCLUSIONS}

Preoperative hyponatremia is common in patients undergoing cardiac surgery. Eighteen percent of patients with normal EF and $35 \%$ of patients with low EF have preoperative hyponatremia. Although hyponatremia is independently associated with adverse outcomes in patients with normal EF, this study failed to demonstrate an association with adverse outcomes in patients with low EF. Despite this lack of association, preoperative hyponatremia should be addressed before surgery regardless of the patient's EF. Further studies are necessary to elucidate the reasons for this unexpected response to hyponatremia in patients with low EF.

\section{References}

1. Upadhyay A, Jaber BL, Madias NE. Incidence and prevalence of hyponatremia. Am J Med. 2006;119:S30-5.

2. Wald R, Jaber BL, Price LL, Upadhyay A, Madias NE. Impact of hospital-associated hyponatremia on selected outcomes. Arch Intern Med. 2010;170:294-302.

3. Gheorghiade M, Abraham WT, Albert NM, Gattis-Stough W, Greenberg BH, O'Connor CM, OPTIMIZE-HF Investigators and Coordinators. Relationship between admission serum sodium concentration and clinical outcomes in patients hospitalized for heart failure: an analysis from the OPTIMIZE-HF registry. Eur Heart J. 2007;28:980-8.

4. Gheorghiade M, Rossi JS, Cotts W, Shin DD, Hellkamp AS, Piña IL, et al. Characterization and prognostic value of persistent hyponatremia in patients with severe heart failure in the ESCAPE Trial. Arch Intern Med. 2007; 167:1998-2005.

5. Klein L, O'Connor CM, Leimberger JD, Gattis-Stough W, Piña IL, Felker GM, et al., OPTIME-CHF Investigators. Lower serum sodium is associated with increased short-term mortality in hospitalized patients with worsening heart failure: results from the Outcomes of a Prospective Trial of Intravenous Milrinone for Exacerbations of Chronic Heart Failure (OPTIME-CHF) study. Circulation. 2005;111:2454-60.

6. Crestanello JA, Phillips G, Firstenberg MS, Sai-Sudhakar C, Sirak J, Higgins R, et al. Preoperative hyponatremia predicts outcomes after cardiac surgery. J Surg Res. 2012 Jun 22. [Epub ahead of print]

7. Hannan EL, Racz MJ, Jones RH, Gold JP, Ryan TJ, Hafner JP, et al. Predictors of mortality for patients undergoing cardiac valve replacements in New York State. Ann Thorac Surg. 2000;70:1212-8.

8. Jamieson WR, Edwards FH, Schwartz M, Bero JW, Clark RE, Grover FL. Risk stratification for cardiac valve replacement. National Cardiac Surgery Database. Ann Thorac Surg. 1999;67:943-51.

9. Appoo J, Norris C, Merali S, Graham MM, Koshal A, Knudtson ML, et al. Longterm outcome of isolated coronary artery bypass surgery in patients with severe left ventricular dysfunction. Circulation. 2004;110(11 Suppl 1):II13-7.

10. Topkara VK, Cheema FH, Kesavaramanujam S, Mercando ML, Cheema AF, Namerow PB, et al. Coronary artery bypass grafting inpatients with low ejection fraction. Circulation. 2005;112(9 Suppl):I344-50.

11. Society of Thoracic Surgeons Adult Cardiac Surgery Database v2.61 Training Manual. Available at: http://www.sts.org/sites/default/files/documents/pdf/ trainingmanuals/adult2.61/Section_O_POSTOPERATIVE.pdf. Accessed December $16,2011$.

12. Society of Thoracic Surgeons Adult Cardiac Surgery Data Specifications v2.61. Available at: http://www.sts.org/sites/default/files/documents/pdf/AdultCVData Specifications2.61.pdf. Accessed December 16, 2011.

13. Hosmer DW, Lemeshow S, May S. Applied Survival Analysis: Regression Modeling of Time to Event Data. 2nd ed. New York, NY: John Wiley and Sons, Inc; 2008.

14. Hosmer DW, Lemeshow S. Applied Logistic Regression. 2nd ed. New York, NY: John Wiley and Sons, Inc; 2000.

15. Schou M, Valeur N, Torp-Pedersen C, Gustafsson F, Kluber L. Plasma sodium and mortality risk in patients with myocardial infarction and a low LVEF. Eur J Clin Invest. 2011;41:1237-44.

16. Rusinaru D, Buiciuc O, Leborgne L, Slama M, Massy Z, Tribouilloy C. Relation of serum sodium level to long-term outcome after a first hospitalization for heart failure with preserved ejection fraction. Am J Cardiol. 2009;103:405-10.

17. Sica DA. Hyponatremia and heart failure-treatment considerations. Congest Heart Fail. 2006;12:55-60.

18. Colucci W, Braunwald E. Pathophysiology of heart failure. In: Braunwald E, Zipes D, Libby P, eds. Heart Disease. Philadelphia, PA: WB Saunders Co; 2001:503-33.

19. Milo-Cotter O, Cotter G, Weatherley BD, Adams KF, Kaluski E, Uriel N, et al. Hyponatraemia in acute heart failure is a marker of increased mortality but not when associated with hyperglycaemia. Eur J Heart Fail. 2008;10:196-200. 


\section{APPENDIX E1. OUTCOMES DEFINITIONS}

Early mortality was defined as any death that occurred during the hospitalization in which the operation was performed or after discharge from the hospital, but within 30 days of the procedure.

Late mortality included any death that occurred both after discharge from the hospital and more than 30 days from the operative procedure.

\section{Complications Defined by the Society of Thoracic Surgeons}

Operative complications included (a) perioperative myocardial infarction; (b) reoperation for bleeding, cardiac tamponade, native or prosthetic valve dysfunction, and graft occlusion; (c) other cardiac reoperations; and (d) noncardiac reoperations.

Pulmonary complications included (a) reintubation during hospital stay, (b) ventilatory support for more than 24 hours after surgery, (c) pulmonary embolism, and (d) pneumonia.

Infectious complications included (a) wound infections (sternal, thoracotomy, leg, and arm incisions) and (b) sepsis.

Neurologic complications included (a) stroke, (b) transient ischemic attack, (c) comma, and (d) paralysis or paraplegia.

Renal complications included renal failure defined as a postoperative serum creatinine greater than $2 \mathrm{mg} / \mathrm{dL}$, or twice the preoperative creatinine level, or the need for renal replacement therapy.

Other complications included (a) cardiac arrest, (b) tamponade, (c) multisystem organ failure, and (d) atrial fibrillation.

\section{APPENDIX E2. COVARIATES INCLUDED IN MULTIVARIABLE REGRESSION ANALYSIS MODELS}

1. Logistic operative complication model: European System for Cardiac Operative Risk Evaluation (euroSCORE), inotropic medications, previous cardiac surgery, cardiogenic shock, coronary artery bypass grafting (CABG), valve surgery, stroke, surgery status (elective, emergency, or urgent), glomerular filtration rate (GFR), and New York Heart Association (NYHA) class.

2. Logistic infectious complication model: euroSCORE, inotropic medications, cardiogenic shock, intra-aortic balloon pump (IABP), arrhythmia, CABG, valve surgery, other cardiac surgery, stroke, previous myocardial infarction (MI), surgery status (elective, emergency, or urgent), GFR, and NYHA classification.

3. Logistic pulmonary complication model: euroSCORE, inotropic medications, IABP, arrhythmia, cardiogenic shock, CABG, valve, ventricular assist device (VAD), stroke, chronic obstructive pulmonary disease (COPD), surgery status (elective, emergency, or urgent), GFR, and NYHA class.

4. Logistic neurologic complication model: euroSCORE, patient age, gender, previous cardiac surgery, inotropic medications, IABP, diabetes, cardiogenic shock, arrhythmia, CABG, valve surgery, VAD surgery, other cardiac surgery, stroke, peripheral vascular disease (PVD), previous MI, hypertension, surgery status (elective, emergency, or urgent), GFR, and NYHA class.

5. Logistic renal failure requiring dialysis model: euroSCORE, previous cardiac surgery, inotropic medications, cardiogenic shock, arrhythmia, CABG, valve surgery, VAD surgery, stroke, previous MI, surgery status (elective, emergency, or urgent), GFR, and NYHA class.

6. Logistic other complication model: euroSCORE, patient age, gender, previous cardiac surgery, inotropic medication, IABP, diabetes, cardiogenic shock, arrhythmia, CABG, valve surgery, VAD surgery, stroke, PVD, previous MI, COPD, surgery status (elective, emergency, or urgent), prior GFR, and NYHA class.

7. Linear length of hospital stay model: euroSCORE, inotropic medications, cardiogenic shock, arrhythmia, CABG, valve surgery, VAD surgery, stroke, surgery status (elective, emergency, or urgent), GFR, and NYHA class.

8. Logistic early mortality model: patient age, GFR, euroSCORE, preoperative inotropic medications, VAD surgery, other cardiac surgery, cardiogenic shock, and NYHA class.

9. Cox proportional hazard late mortality model: patient age, GFR, euroSCORE, preoperative inotropic medications, VAD surgery, other cardiac surgery, cardiogenic shock, and NYHA classification.

10. Cox proportional hazard overall mortality model: patient age, GFR, euroSCORE, preoperative inotropic medications, VAD surgery, cardiogenic shock, and surgery status (elective, emergency, or urgent). 


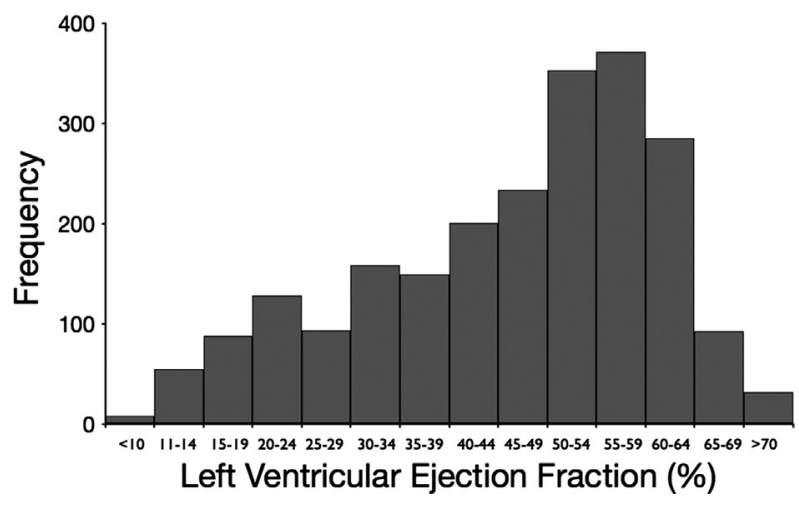

FIGURE E1. Distribution of preoperative LV EF. Low EF was present in 707 patients $(31.5 \%)$ ). A total of 550 patients $(24.5 \%)$ had moderate reduction of $\mathrm{EF}$ (EF, $20 \%-39 \%$ ). A total of 157 patients (7.0\%) had severe reduction of $\mathrm{EF}(<20 \%)$. $L V$, Left ventricular; $E F$, ejection fraction.

\section{Low EF}

Complications:

Operative, OR $(95 \% \mathrm{Cl}) \quad 2.06(1.37-3.09)$

Infectious, OR $(95 \% \mathrm{Cl}) \quad 1.19(0.67-2.14)$

Pulmonary, OR (95\% Cl) $2.14(1.56-2.96)$

Neurologic, OR $(95 \% \mathrm{Cl}) \quad 1.76(0.77-4.06)$

Renal failure, OR $(95 \% \mathrm{Cl}) \quad 1.93(1.19-3.12)$

Dialysis, OR (95\% Cl) $1.98(1.10-3.57)$

Other, OR $(95 \% \mathrm{Cl}) \quad 1.02(0.72-1.43)$

Hospital stay, multiplier $(95 \% \mathrm{Cl}) \quad 1.47(1.32-1.64)$

Early mortality, OR $(95 \% \mathrm{Cl})$

Late mortality, $\mathrm{HR}(95 \% \mathrm{Cl})$

$2.01(1.18-3.42)$

$1.34(0.96-1.87)$

$1.48(1.12-1.95)$

Overall mortality, $\mathrm{HR}(95 \% \mathrm{Cl})$
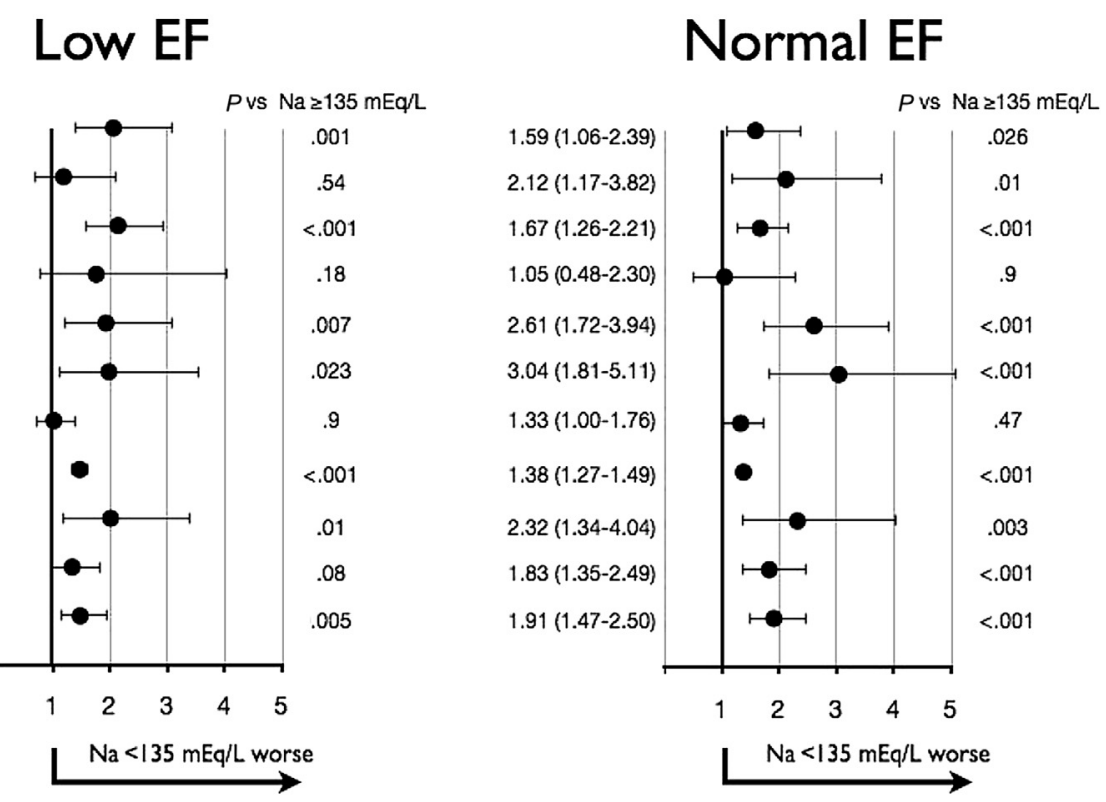

FIGURE E2. Effect of hyponatremia on clinical outcomes of patients with low and normal ejection fractions by univariate analysis. EF, Ejection fraction; $O R$, odds ratio; $C I$, confidence interval; $H R$, hazard ratio. 